likely to undergo colectomy (HR 1.94, 95\% CI: 1.59-2.37, p < 0.001). 5-ASA use was protective, with users $65 \%$ less likely to undergo colectomy than non-users (HR 0.35 , 95\% CI: $0.28-$ $0.44, \mathrm{p}<0.001)$.

Conclusion Male sex, TP use and early steroid use within 3 months of diagnosis are predictors of colectomy in UC. Amongst TP users, sustained TP use for greater than 12 months duration, was associated with a reduction in colectomy rates. 5-ASA use was associated with a two thirds reduction in risk of colectomy. Our findings support the role of 5-ASA use and prolonged TP treatment for UC patients with a severe disease phenotype.

Disclosure of Interest None Declared.

\section{PWE-119 DECREASED FREQUENCY OF PERIPHERAL AND INTESTINAL NKG2A-POSITIVE T CELLS IN ULCERATIVE COLITIS PATIENTS}

${ }^{1}$ W Kobayashi*, ${ }^{1}$ T Katsurada, ${ }^{2} \mathrm{U}$ Tomaru, ${ }^{1} \mathrm{~N}$ Sakamoto, ${ }^{2} \mathrm{M}$ Kasahara. ${ }^{1}$ Gastroenterology, Hokkaido University Graduate School of Medicine, Sapporo, Japan; ${ }^{2}$ Pathology, Hokkaido University Graduate School of Medicine, Sapporo, Japan

\subsection{6/gutjnl-2014-307263.379}

Introduction The intestinal tract is home to a numerous immune cellular components that continuously encounter abundant exogenous stimuli, where remain in a state of controlled inflammation normally. Inflammatory bowel disease (IBD) is characterised by chronic and refractory intestinal inflammation complising ulcerative colitis (UC) and Crohn's disease (CD) in main. Inhibitory natural killer receptors (iNKRs) are expressed on NK cells, involved in NK-cell tolerance to self. There is increasing evidence that iNKRs like NKG2A expressed on T cells are importantly involved in the regulation of immune responses though no studies have addressed the potential role in the pathogenesis of IBD. We analysed the expression of NKG2A on T cells in dextran sulfate sodium (DSS)-induced colitis model mouse and IBD patients.

Methods Male BALB/c mice were administrated 5\% DSS in distilled water for 7 days ad libitum for induction of experimental colitis. For controls, age-matched $\mathrm{BALB} / \mathrm{c}$ mice were given distilled water. NKG2A $+\mathrm{T}$ cells in peripheral blood mononuclear cells (PBMCs) and lamina propria mononuclear cells (LPMCs) were analysed by flow cytometry. For histological analysis of DSS-induced colitis mice, hematoxylin and eosin (HandE) staining and immunohistochemistry were performed. For blocking experiments using antibody (Ab), mice were intraperitoneally injected with $300 \mu \mathrm{g}$ of anti-NKG2A monoclonal $\mathrm{Ab}$ or control $\mathrm{IgG}$ after oral administration of $5 \%$ DSS. PBMCs samples from 23 healthy controls, 20 UC and $16 \mathrm{CD}$ patients were analysed by flow cytometry. Tissue sections from 7 controls, $6 \mathrm{UC}$ and $5 \mathrm{CD}$ patients were subjected to histological analysis. Data were evaluated using the Student's $t$-test or the Mann-Whitney U-test.

Results In the active phase of DSS-induced colitis mouse, the frequency of NKG2A $+\mathrm{T}$ cells was significantly decreased in the peripheral blood and increased in the intestine, suggesting the mobilisation of this $\mathrm{T}$ cell subset to the sites of inflammation. Administration of anti-NKG2A Ab increased the number of inflammatory foci in DSS-induced colitis, suggesting the involvement of NKG2A+ T cells in this colitis model. In UC patients, the frequency of NKG2A $+\mathrm{T}$ cells in PBMCs was significantly decreased, compared with $\mathrm{CD}$ patients and healthy controls, regardless of clinical conditions. Notably, in sharp contrast to the DSS-induced colitis mouse, the frequency of NKG2A+ cells in LPMCs was also decreased in UC patients.

Conclusion These results suggest that inadequate local infiltration of NKG2A $+\mathrm{T}$ cells may be involved in the pathogenesis of UC. Our study demonstrates the frequency of NKG2A+ T cells is decreased in both PBMCs and LPMCs in UC patients, implicating this $\mathrm{T}$ cell subset as a potential therapeutic target for UC.

Disclosure of Interest None Declared.

\section{PWE-120 WHAT DO HEALTHCARE PROFESSIONALS KNOW ABOUT FATIGUE IN PATIENTS WITH IBD AND HOW DO THEY MANAGE IT?}

${ }^{1}$ WJ Czuber-Dochan*, ${ }^{1} \mathrm{C}$ Norton, ${ }^{2} \mathrm{~S}$ Berliner, ${ }^{3} \mathrm{~F}$ Bredin, ${ }^{2} \mathrm{M}$ Darvell, ${ }^{4} \mathrm{~A}$ Forbes, ${ }^{2} \mathrm{M}$ Gay, ${ }^{4} \mathrm{I}$ Nathan, ${ }^{1} \mathrm{E}$ Ream, ${ }^{2} \mathrm{H}$ Terry. ${ }^{1}$ Florence Nightingale School of Nursing and Midwifery, King's College London, London, UK; ${ }^{2}$ Crohn's and Colitis UK, St Albans, UK; ${ }^{3}$ Gastroenterology, The Queen Elizabeth Hospital NHS Trust, King's Lynn, UK; ${ }^{4}$ Gastroenterology and Clinical Nutrition, University College London, London, UK

\subsection{6/gutjnl-2014-307263.380}

Introduction Fatigue is one of the top complaints in inflammatory bowel disease (IBD) with $40 \%$ of patients in remission and $86 \%$ in active condition reporting fatigue. ${ }^{1}$ However patients report that their complaints of fatigue are often not addressed in clinical consultations. ${ }^{2}$ This study aimed to gain an understanding of healthcare practitioners' (HCPs) perception of IBD fatigue as experienced by people with IBD.

Methods Descriptive phenomenology with purposive sampling was used to identify a range of professionals (gastroenterologists, IBD nurses, general practitioners, dietitians, psychologists and pharmacists). In-depth semi-structured interviews were conducted with 20 HCPs who work with people with IBD (June-Dec 2012). Colazzi's framework was used to analyse the data. $^{3}$

Results Three main themes and several sub-themes were identified. The main themes were: the phenomenon of fatigue as perceived by HCPs; the impact of fatigue on patients' lives; and the methods used by HCPs to deal with fatigue. Fatigue was identified as an important, but difficult and often frustrating, symptom to understand. The study participants perceived fatigue as 'a complicated and complex thing'. HCPs reported that fatigue impacts on the emotional, private and public aspects of patients' functioning, however there were very few methods suggested on how to assess and manage the fatigue in a systematic way. Many expressed a desire for better education about fatigue and better multi-disciplinary effort to manage fatigue.

Conclusion Despite fatigue being one of the symptoms most frequently reported by IBD patients, it remains poorly understood by HCPs, who find fatigue challenging and frustrating. There is a need for a systematic and structured assessment and management of this distressing symptom and HCPs should communicate with each other about care for each individual patient. There is a need for an assessment framework and for intervention strategies to be tested. It is essential for multidisciplinary team members to be involved in planning and managing coordinated care of patients reporting fatigue in IBD.

\section{REFERENCES}

1 Czuber-Dochan W, Ream E, Norton C, Review article: description and management of fatigue in inflammatory bowel disease. Alim Pharma and Therap 2013;37(5):505-16 